\title{
Efeito anti-helmíntico do hidrolato de Mentha villosa Huds. (Lamiaceae) em nematóides gastrintestinais de bovinos
}

\author{
Anthelmintic effect of hidrolact of Mentha villosa Huds. (Lamiaceae) in gastrointestinal \\ nematodes of cattle
}

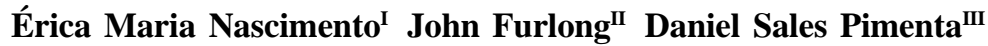 \\ Márcia Cristina de Azevedo Prata ${ }^{\mathrm{II}}$
}

\section{RESUMO}

Atualmente, o estudo da atividade anti-helmíntica de plantas medicinais em ruminantes tem atraído bastante interesse. Mentha villosa Huds. (Lamiaceae) é uma das espécies de hortelã que tem sido utilizada popularmente devido às diversas propriedades medicinais, inclusive para o controle de verminoses. O presente estudo teve como objetivo testar a atividade anti-helmíntica do hidrolato dessa planta em bezerras infectadas por nematóides gastrintestinais, tanto in vitro, pelo método de coprocultura quantitativa, quanto in vivo, por meio do teste de redução no número de ovos de nematóides nas fezes dos hospedeiros. No teste in vitro, o hidrolato nas concentrações de 40\%, 60\% e 80\% e 100\% apresentou porcentagem de eficácia de 91,88\%, 94,15\%, 98,40\% e 100\%, respectivamente, mostrando atividade ovicida significativa sobre nematóides gastrintestinais em bezerras. Entretanto, os resultados do teste in vivo mostraram ausência de atividade anti-helmíntica do hidrolato de $\mathbf{M}$. villosa na dose de $0,1 \mathrm{ml} \mathrm{kg}$ dia $^{-1}$, nos animais tratados.

Palavras-chave: plantas medicinais, fitoterapia, helmintos, hortelã, Mentha villosa.

\section{ABSTRACT}

Currently, it has been of great interest to study the anthelmintic activity of medicinal plants in ruminants. Mentha villosa Huds. (Lamiaceae) is one of the mint species that has been popularly used based on various medicinal properties, even for the control of nematode infections. This study aimed to test the anthelmintic activity of hidrolact of this plant, both in vitro, by the quantitative coproculture method, and in vivo, in calves infected with gastrointestinal nematodes, through the egg count reduction test in feces of the hosts. In in vitro tests, the hidrolact at the concentrations $40 \%, 60 \%$ and $80 \%$ and $100 \%$ obtained percentage of effectiveness of $91.88 \%, 94.15 \%$, $98.40 \%$ and $100 \%$ respectively, showing significant ovicidal activity against gastrointestinal nematodes in calves. However, the hidrolact of $\mathbf{M}$. villosa showed no in vivo anthelmintic activity at $0.1 \mathrm{ml} \mathrm{kg}^{-1}$ day $^{-1}$ on the treated animals.

Key words: medicinal plants, phytotherapy, helminths, mint, Mentha villosa.

\section{INTRODUÇÃO}

O controle das helmintoses vem sendo realizado por meio do uso de anti-helmínticos sintéticos. Entretanto, esse procedimento apresenta alguns problemas, como altos custos, perigo de contaminação ambiental e preocupação do consumidor com relação aos resíduos das drogas nos produtos de origem animal, tais como carne e leite (CHAGAS, 2004; CAMURÇA-VASCONCELOS, 2006). Além disso, segundo SOUZA et al. (2008), o uso intensivo de antihelmínticos, subdoses, diagnósticos incorretos e a falta de rotatividade de bases farmacológicas têm provocado um sério problema sanitário, que é a resistência de nematóides aos fármacos.

Por representar uma alternativa sustentável à quimioterapia convencional, o uso de plantas

\footnotetext{
IPrograma de Pós-graduação em Ciências Biológicas, Comportamento e Biologia Animal, Instituto de Ciências Biológicas, Universidade Federal de Juiz de Fora (UFJF), Bairro Martelos, s/nº, 36036-900, Juiz de Fora, MG, Brasil. E-mail: ericamjf@yahoo.com.br.*Autor para correspondência.

IEmbrapa Gado de Leite, Juiz de Fora, MG, Brasil.

IIIDepartamento de Botânica, Instituto de Ciências Biológicas, UFJF, Juiz de Fora, MG, Brasil.
} 
medicinais e o interesse em suas propriedades antiparasitárias têm aumentado, conforme demonstrado em várias revisões na área de medicina veterinária (CHAGAS, 2004; FURTADO, 2006; GITHIORI et al., 2006; ATHANASIADOU et al., 2007). Segundo ANTHONY et al. (2005), os óleos essenciais de plantas (e/ou os princípios ativos) podem ser utilizados como alternativas ou aliados às terapias antiparasitárias convencionais.

Alguns estudos etnofarmacológicos têm relatado que plantas do gênero Mentha L. (Lamiaceae) têm atividade antiparasitária, incluindo Mentha villosa Huds., popularmente conhecida como hortelã-comum, hortelã-de-tempero, hortelã-rasteira ou mentrasto (RADÜNZ, 2004). Tem sido relatado o uso dessa espécie contra amebíases, giardíases, tricomoníases urogenitais e esquistossomose, dentre outras atividades farmacológicas (SOUSA et al., 1997; MONTE \& OLIVEIRA, 2001; LAHLOU et al., 2002). A adição de leite às folhas trituradas de $\boldsymbol{M}$. villosa é utilizada para o tratamento de verminoses em crianças (MEDEIROS et al., 2004). Alguns autores detectaram a presença de óxido de piperitenona (rotundifolona) como constituinte majoritário no óleo essencial de $\boldsymbol{M}$. villosa. No entanto, não foi possível determinar se seria este ou outro o componente que age como seu princípio ativo antiparasitário (RADÜNZ, 2004; ARRUDA et al., 2006; MARTINS et al., 2007).

Este estudo teve como objetivo avaliar o efeito in vitro do hidrolato de $\boldsymbol{M}$. villosa sobre o desenvolvimento de ovos de nematóides gastrintestinais de bovinos, por meio da técnica de coprocultura quantitativa, bem como testar o efeito antihelmíntico in vivo do hidrolato de $\boldsymbol{M}$. villosa em bezerras mestiças (Holandês x Zebu) infectadas por nematóides gastrintestinais, por meio do teste de diminuição na contagem de ovos de nematóides gastrintestinais nas fezes.

\section{MATERIAL E MÉTODOS}

Amostras do ramo e das folhas de $\boldsymbol{M}$. villosa foram coletadas às 8h, em março de 2007, na Estação de Cultivo e Manutenção de Plantas da Universidade Federal de Juiz de Fora. Exsicatas foram depositadas no Herbário Leopoldo Krieger da UFJF sob o número de registro CESJ-48632. Após a planta ter sido acondicionada em congelador $\left(-18^{\circ} \mathrm{C}\right)$ por 20 dias, foi extraído o hidrolato (caracterizado como a fração aquosa contendo o óleo essencial emulsionado), pelo método de hidrodestilação em aparelho Clevenger, quando a planta (100g de material fresco) permaneceu em ebulição por duas horas em $1 \mathrm{l}$ de água. Foram preparadas as seguintes soluções com adição de água destilada: hidrolato $100 \%$, hidrolato $80 \%$, hidrolato $60 \%$, hidrolato $40 \%$ e hidrolato $20 \%$. Essas soluções foram mantidas em frascos no congelador $\left(-18^{\circ} \mathrm{C}\right)$ até a realização dos testes, quando foram agitadas para proporcionar sua emulsão.

Para a realização do teste in vitro, foram obtidas amostras de fezes contaminadas naturalmente com ovos de nematóides gastrintestinais, sendo estas coletadas diretamente da ampola retal de bezerras mestiças (Holandês x Zebu) criadas no Campo Experimental de Santa Mônica/Embrapa Gado de Leite, localizado no Município de Valença, Rio de Janeiro (RJ). As fezes foram mantidas em sacos plásticos sob refrigeração (aprox. $5^{\circ} \mathrm{C}$ ) e enviadas ao Laboratório de Parasitologia da Embrapa Gado de Leite em Juiz de Fora, Minas Gerais (MG), onde foi realizada a contagem de ovos por grama de fezes (opg) em Câmara de McMaster, de acordo com a técnica modificada de GORDON \& WHITLOCK (1939), citada em UENO \& GONÇALVES (1998), para verificação do nível de contaminação.

Em seguida, foi realizado teste para avaliação do efeito do hidrolato de $\boldsymbol{M}$. villosa sobre a eclosão de larvas de nematóides gastrintestinais. Os grupos testados consistiram em: A - controle negativo, no qual foi utilizada água destilada; B - controle positivo, em que foi usado o anti-helmíntico comercial Albendazol; C - grupo de tratamento, com a utilização do hidrolato em cinco concentrações (20\%, 40\%, 60\%, $80 \%$ e $100 \%$ ), sendo feitas 30 repetições para cada tratamento.

O cultivo de larvas de nematóides gastrintestinais foi realizado pelo método de coprocultura quantitativa, adaptado de UENO \& GONÇALVES (1998) e inicialmente descrito por ROBERTS \& O’SULLIVAN (1950). O líquido obtido, contendo as larvas L3, foi colocado em tubos de ensaio com capacidade para $15 \mathrm{ml}$. Após centrifugação por 10 minutos a 1.500rpm, foi mantido o líquido contendo as larvas com dois cm de profundidade em todos os tubos de ensaio, sendo descartado o restante. As amostras foram então mantidas em refrigerador $\left(10^{\circ} \mathrm{C}\right)$ por, no máximo, 30 dias, até a contagem das larvas ao microscópio em lâminas temporárias, utilizando-se aproximadamente $3 \mathrm{ml}$ do conteúdo líquido previamente homogeneizado. As larvas foram identificadas utilizando-se o Manual para Diagnóstico das Helmintoses de Ruminantes elaborado por UENO \& GONÇALVES (1998).

Para a determinação da porcentagem de eficácia das substâncias testadas, foi empregada a seguinte fórmula:

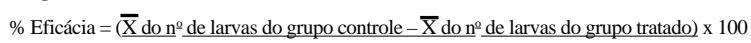
$\overline{\mathrm{X}}$ do no de larvas do grupo controle 
Foram empregados os testes nãoparamétricos Kruskal-Wallis e Dunn com níveis de significância de 5\% (Programa Graph Pad Instat ${ }^{\mathrm{tm}}$ ) para verificar a existência de diferenças significativas entre os grupos experimentais, já que a amostra não apresentou distribuição normal, de acordo com o teste de Barlett.

O teste anti-helmíntico in vivo foi conduzido no Campo Experimental de Santa Mônica em Valença, RJ. Foram utilizadas bezerras mestiças (Holandês $x$ Zebu), entre oito e 10 meses de idade e peso médio de $137,9 \mathrm{~kg}( \pm 28,2 \mathrm{~kg})$. Os animais utilizados não receberam qualquer tipo de tratamento anti-helmíntico nas 12 semanas antecedentes ao início do experimento e apresentavam sua própria identificação, que consistiu em brincos plásticos aplicados na orelha e tatuagem, além de cordas de nylon coloridas no pescoço para identificação de cada grupo experimental.

Tendo em vista o nível de infecção insuficiente para a realização dos testes, verificado por análises periódicas de opg nos animais, foi realizada uma infecção artificial três meses antes do início dos tratamentos. Para tal, foi realizado o cultivo de larvas de nematóides gastrintestinais pelo método de coprocultura quantitativa a partir de amostras fecais. As larvas L3 obtidas foram acondicionadas, juntamente com 600ml de água destilada em frasco mantido sob refrigeração (aprox. $5^{\circ} \mathrm{C}$ ), sendo administrada dose de 5000 larvas oralmente a cada uma das 24 bezerras por meio de uma seringa descartável.

O teste anti-helmíntico in vivo teve início três meses após a infecção artificial, período no qual foram feitas análises periódicas de fezes dos animais infectados até que se atingisse um nível de opg suficiente para a realização dos testes. Três dias antes do início do teste (dia -3), os animais foram pesados em balança eletrônica, e três dias antes do início dos tratamentos (dias -3 a -1) foi realizada a contagem de ovos por grama de fezes, diariamente, às $7 \mathrm{~h}$.

A contagem de opg foi realizada a partir da adição de 54ml de solução hipersaturada de açúcar a $6 g$ de fezes, de acordo com técnica descrita em UENO \& GONÇALVES (1998). A partir da contagem de ovos realizada (média entre os três dias), foram formados três grupos experimentais, a saber: 1- grupo controle negativo (água destilada); 2- grupo de tratamento (hidrolato a 100\% de Mentha villosa); 3- grupo controle positivo (anti-helmíntico sintético Ricobendazole ${ }^{\circledR}$ ). A administração das substâncias dos grupos 1 e 2 foi realizada uma vez ao dia, sempre entre 7 h30 e 8 h, durante 10 dias consecutivos (dia 0 ao dia +9 ), sendo feitas oralmente por meio de uma seringa descartável inserida no canto da boca dos animais, para evitar o fechamento da goteira esofágica. No grupo 1 ( $\mathrm{n}=6$ animais), foram administrados $15 \mathrm{ml}$ de água destilada igualmente para todos os animais. Os animais do grupo $2(n=5)$ receberam hidrolato na dose de $0,1 \mathrm{ml} \mathrm{kg}^{-1} \mathrm{dia}^{-1}$. Nos animais do grupo $3(n=6)$, foi aplicado o antihelmíntico Ricobendazole ${ }^{\circledR}$ (sulfóxido de albendazol, veículo q.s.p. - Ourofino) no primeiro dia de tratamento (dia 0), por via subcutânea, no pescoço dos animais, em dose única de $1 \mathrm{ml} 40 \mathrm{~kg}^{-1}$, com auxílio de pistola dosificadora, conforme indicado pelo fabricante.

Antes da realização dos tratamentos (dias 3 a -1), foram coletadas amostras de fezes das 17 bezerras para realização de nova contagem de opg, utilizando-se a técnica de solução de açúcar descrita anteriormente. Essas coletas foram realizadas diariamente durante 10 dias (dia +1 ao dia +10) e também uma e duas semanas após o final dos tratamentos (dias +17 e +24). Parte da amostra de fezes da última coleta dos animais do grupo 1 foi utilizada para obtenção de larvas L3, por meio de coprocultura, com o objetivo identificar e quantificar as larvas presentes. As larvas foram identificadas utilizando-se o Manual para Diagnóstico das Helmintoses de Ruminantes elaborado por UENO \& GONÇALVES (1998).

Durante o período de administração dos tratamentos e coleta de fezes, as bezerras foram mantidas em jejum, sempre das $16 \mathrm{~h}$ do dia anterior até o momento em que foram tratadas, sendo então transferidas para piquetes contendo pastagem de capim elefante (Pennisetum purpureum, Schum), além de ração ( $2 \mathrm{~kg}$ animal $^{-1}$ dia $\left.^{-1}\right)$ e água ad libitum.

Devido à ocorrência de alto desvio padrão quanto à contagem de opg antes do tratamento entre os animais dos grupos, bem como entre os grupos experimentais, não foi possível realizar comparações entre o grupo tratado e os grupos de controle. Desse modo, a porcentagem de eficácia do hidrolato testado foi determinada com base na contagem de opg feita durante o tratamento e no pós-tratamento (dias $0 \mathrm{a}+9$, +17 e +24), tendo como base o período de prétratamento (média entre os dias -3, -2, e -1). Para isso, foi empregada a seguinte fórmula:

$\%$ Eficácia do hidrolato (dia $\mathrm{x})=(\underline{\bar{X} \text { de opg dos dias }-3 \mathrm{a}-1-\overline{\mathrm{X}} \text { de opg do dia } \mathrm{x})} \times 100$ $\bar{X}$ de opg dos dias -3 a -1

\section{RESULTADOS E DISCUSSÃO}

O hidrolato de $\boldsymbol{M}$. villosa apresentou o efeito ovicida sobre nematóides gastrintestinais de bovinos no teste in vitro, sendo esse efeito dose-dependente. As concentrações de hidrolato a $80 \%$ e $100 \%$ mostraram eficácia acima de $98 \%(98,40 \%$ e $100 \%$, respectivamente), valor considerado altamente efetivo segundo o Ministério da Agricultura, Pecuária e 
Abastecimento, de acordo com as recomendações contidas na Portaria no 48, de 12 de maio de 1997. As concentrações de hidrolato entre $40 \%$ e $60 \%$ apresentaram-se efetivas. Somente o hidrolato a $20 \%$ apresentou-se insuficientemente ativo (66,44\%). Já o hidrolato a $100 \%$, mostrou máxima eficácia, sendo esta equivalente ao controle positivo utilizando o antihelmíntico Albendazol ${ }^{\circledR}$ (Tabela 1, Figura 1). KETZIS et al. (2006), por outro lado, afirmam que um critério de eficácia único (por exemplo, 90\% de redução da carga parasitária) não pode ser aplicado a novos métodos de controle, sendo necessária maior tolerância em comparação com os anti-helmínticos comerciais.

Quanto aos nematóides presentes nas amostras analisadas, observou-se predominância dos gêneros Cooperia e Haemonchus (cerca de 90\% das larvas encontradas), além de larvas dos gêneros Oesophagostomum e Trichostrongylus em menor número (cerca de 6\% e 4\%, respectivamente).

No teste in vivo, o hidrolato de $\boldsymbol{M}$. villosa, na dose utilizada $\left(0,1 \mathrm{ml} \mathrm{kg}^{-1} \mathrm{dia}^{-1}\right)$, não provocou redução na contagem de opg nas bezerras infectadas por nematóides gastrintestinais (Tabela 2). Pode ocorrer, com certa freqüência, em experimentos que abordem o efeito anti-helmíntico de algumas substâncias, disparidade entre os resultados in vitro e in vivo (FURTADO, 2006; ROCHFORT et al., 2008). Isso também foi observado em outros experimentos (PESSOA, et al., 2002; COSTA, 2004; FURTADO, 2006). Por exemplo, Chenopodium ambrosioides e seu óleo essencial reduziram a viabilidade dos ovos de $\boldsymbol{H}$. contortus em teste in vitro, porém não apresentaram efeito sobre a redução do número de nematóides adultos ou ovos, quando administrada a caprinos (KETZIS et al., 2002). Isso pode ter ocorrido porque consideráveis diferenças fisiológicas são esperadas em condições in vitro e in vivo (GITHIORI et al., 2006). Segundo EGUALE et al (2007), o efeito negativo nos testes in vivo pode ser atribuído à biotransformação de alguns dos componentes ativos dentro do trato digestivo do hospedeiro. O sistema digestivo peculiar dos ruminantes pode mudar o metabolismo ou o modo de ação de alguns nutrientes, medicamentos ou materiais bioativos quando administrados oralmente (VANDAMME \& ELLIS, 2004).

Com relação à metodologia utilizada no teste in vivo, o principal método de detecção de resistência de nematóides a anti-helmínticos sintéticos continua sendo o teste de redução na contagem de ovos nas fezes, que pode ser usado com todos os grupos de anti-helmínticos (RANGEL et al., 2005; COLES et al., 2006). Esse método também tem sido utilizado em outros estudos para analisar a atividade anti-helmíntica de plantas em ruminantes (GITHIORI et al., 2004; FURTADO, 2006; POMROY \& ADLINGTON, 2006) e também é recomendado pelo Ministério da Agricultura, Pecuária e Abastecimento, de acordo a Portaria oㅜ 48, de 12 de maio de 1997. Segundo FURTADO (2006), a redução na contagem dos ovos nas fezes não está diretamente associada a uma mortalidade do parasito, podendo ocorrer apenas supressão na eliminação dos ovos. Porém, esse fato por si só já é um bom recurso no controle parasitário, pois determina diminuição da contaminação da pastagem, que passa a apresentar menor número de larvas infectantes.

As coletas de fezes para realização da contagem de opg no teste in vivo foram feitas em período geralmente utilizado em outros experimentos. COLES et al. (1992) recomendam que as fezes sejam coletadas entre o dia 10 e o dia 14 após o tratamento, pois a análise em tempo inferior poderia gerar resultados equivocados. Outro fator que pode ter influenciado os resultados foi o número de animais utilizados por grupo experimental. VERCRUYSSE et al. (2001) relataram que, em casos de pesquisa, o experimento pode contar com, no mínimo, seis animais por grupo, assim como recomenda a Portaria no 48 do Ministério da Agricultura, Pecuária e Abastecimento, de 12 de maio de 1997. No presente estudo, foram utilizados seis animais nos grupos de controle e cinco no grupo tratado devido à dificuldade em se obterem animais com o mínimo de opg suficiente para a realização do teste, mesmo após realização de infecção artificial. Outros estudos, relativos a teste anti-helmíntico in vivo em ruminantes,

Tabela 1 - Número de larvas de nematóides gastrintestinais obtidas a partir de coprocultura realizada na Embrapa Gado de Leite, Juiz de fora, Minas Gerais (MG), sendo utilizadas amostras de fezes de bezerras contaminadas com ovos de nematóides gastrintestinais, submetidas a tratamento com água destilada (controle negativo), Albendazol ${ }^{\circledR}$ (controle positivo) e hidrolato extraído por hidrodestilação a partir de folhas e ramos de Mentha villosa Huds. testados em cinco concentrações (H 20\%, H 40\%, H 60\%, H 80\%, H 100\%) (n = tamanho da amostra; $\mathrm{x}=$ média; DP = desvio padrão; IC = intervalo de confiança de 95\%).

\begin{tabular}{lccccccc}
\hline & Água destilada & H 20\% & H 40\% & H 60\% & H 80\% & H 100\% & Albendazol $^{\circledR}$ \\
\hline $\mathrm{n}$ & 30 & 30 & 30 & 30 & 30 & 30 \\
$\mathrm{x}+\mathrm{DP}$ & $148,10^{\mathrm{a}} \pm 66,54$ & $49,70^{\mathrm{ab}} \pm 24,45$ & $12,03^{\mathrm{bc}} \pm 12,60$ & $8,67^{\mathrm{c}} \pm 8,10$ & $2,37^{\mathrm{cd}} \pm 2,77$ & $0^{\mathrm{d}}$ & 30 \\
$\mathrm{IC}$ & $123,26-172,94$ & $40,57-58,83$ & $7,33-16,74$ & $5,64-1,69$ & $1,33-3,40$ & - & $0^{\mathrm{d}}$ \\
\hline
\end{tabular}




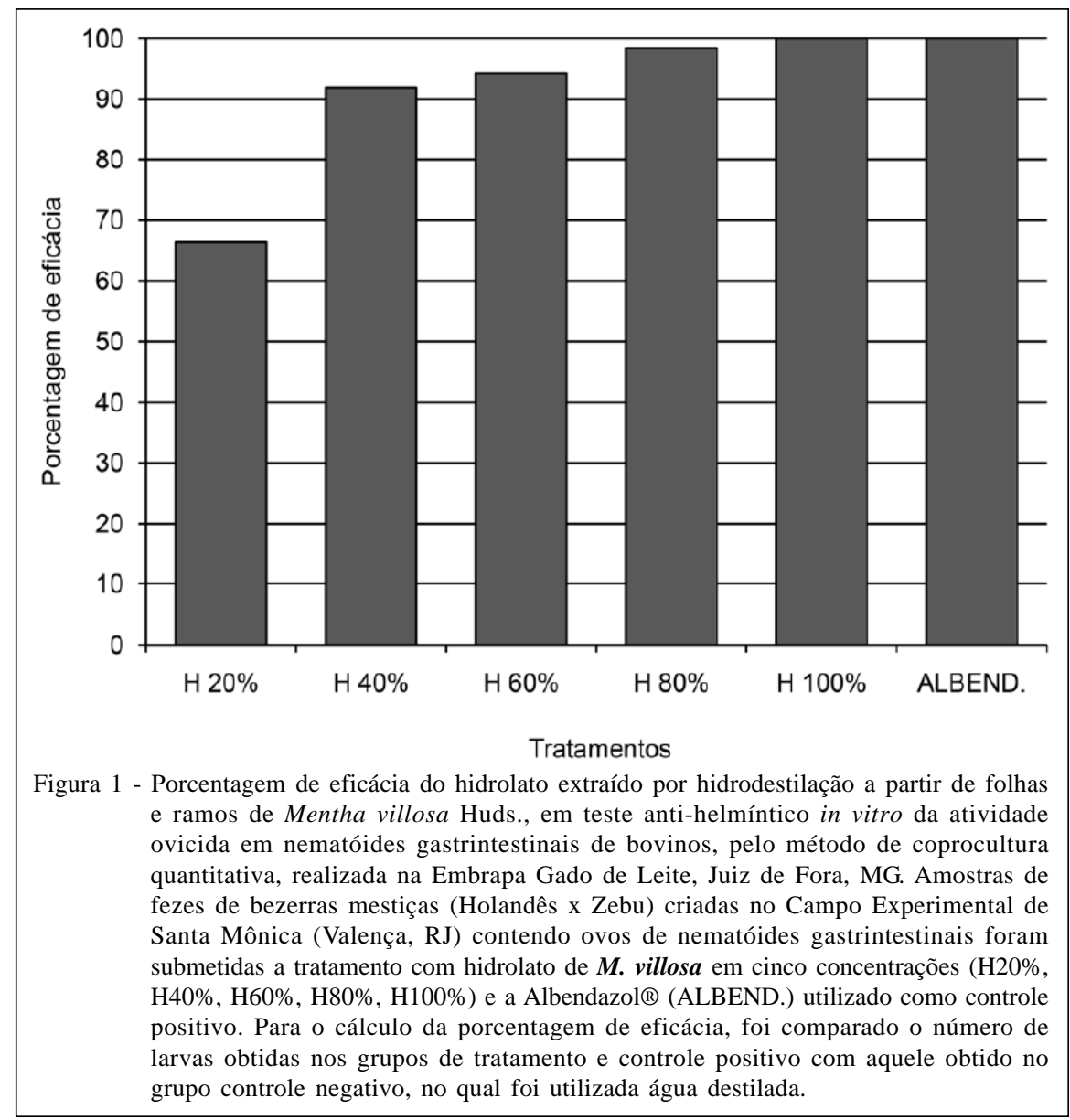

Tabela 2 - Contagem de ovos de nematóides por grama de fezes (opg) provenientes de bezerras mestiças (Holandês x Zebu), infectadas com nematóides gastrintestinais, tratadas com hidrolato extraído por hidrodestilação a partir de folhas e ramos de Mentha villosa Huds. administrados oralmente, durante 10 dias consecutivos (dias $0 \mathrm{a}+9$ ), na dosagem de $1 \mathrm{ml}^{4} \mathrm{~kg}^{-1} \mathrm{dia}^{-1}$ (grupo-tratamento, $\mathrm{n}=5$ ), Ricobendazole ${ }^{\circledR}$ aplicado no dia 0 , por via subcutânea, em dose única de $1 \mathrm{ml} 40 \mathrm{~kg}^{-1}$ (controle positivo, n=6) e água destilada administrada oralmente, durante 10 dias consecutivos (dias 0 a +9 ) (controle negativo, $n=6$ ), e em teste anti-helmíntico in vivo realizado no Campo Experimental de Santa Mônica/ Embrapa Gado de Leite (Valença, RJ). A porcentagem de eficácia do hidrolato de Mentha villosa, calculada com base nos valores de opg obtidos no período de pré-tratamento, também está representada.

\begin{tabular}{|c|c|c|c|c|}
\hline \multirow{2}{*}{ Análise de opg } & \multicolumn{2}{|c|}{------------Hidrolato de $\boldsymbol{M}$. villosa------------ } & \multirow{2}{*}{$\begin{array}{c}\text { Ricobendazole }^{\circledR} \\
\text { (média de opg } \pm \text { desvio padrão) }\end{array}$} & \multirow{2}{*}{$\begin{array}{c}\text { Água destilada } \\
\text { (média de opg } \pm \text { desvio padrão) }\end{array}$} \\
\hline & (média de opg \pm desvio padrão) & Eficácia (\%) & & \\
\hline Dia -1 & $150,26 \pm 68,47$ & $-4,93$ & $272,84 \pm 168,12$ & $270,65 \pm 100,29$ \\
\hline Dia 0 & $157,67 \pm 179,41$ & 6,96 & $226,06 \pm 47,48$ & $357,39 \pm 212,60$ \\
\hline Dia +1 & $139,80 \pm 215,90$ & 11,49 & $12,39 \pm 11,14$ & $241,39 \pm 189,75$ \\
\hline $\mathrm{Dia}+2$ & $133,00 \pm 159,35$ & 8,96 & $0,72 \pm 0,74$ & $244,28 \pm 234,56$ \\
\hline Dia +3 & $136,80 \pm 207,70$ & $-28,04$ & $0,28 \pm 0,25$ & $245,61 \pm 205,25$ \\
\hline Dia +4 & $192,40 \pm 147,84$ & 1,46 & $0,50 \pm 0,46$ & $313,83 \pm 208,79$ \\
\hline Dia +5 & $148,07 \pm 130,30$ & 9,8 & $0,44 \pm 0,40$ & $301,94 \pm 202,08$ \\
\hline Dia +6 & $135,53 \pm 186,13$ & $-77,65$ & $0,11 \pm 0,17$ & $373,11 \pm 281,06$ \\
\hline Dia +7 & $266,93 \pm 411,65$ & 11,35 & $0,72 \pm 0,49$ & $267,72 \pm 219,54$ \\
\hline Dia +8 & $133,20 \pm 139,06$ & $-131,11$ & $0,11 \pm 0,27$ & $227,11 \pm 211,75$ \\
\hline Dia +9 & $347,27 \pm 297,58$ & $-81,69$ & $0,17 \pm 0,28$ & $250,50 \pm 168,91$ \\
\hline Dia +10 & $273,00 \pm 385,25$ & $-273,31$ & $0,11 \pm 0,27$ & $186,83 \pm 130,07$ \\
\hline Dia +17 & $560,93 \pm 701,22$ & $-175,61$ & $0,50 \pm 0,75$ & $374,61 \pm 348,39$ \\
\hline Dia +24 & $414,13 \pm 556,93$ & & $127,89 \pm 287,52$ & $249,83 \pm 231,07$ \\
\hline
\end{tabular}

Ciência Rural, v.39, n.3, mai-jun, 2009. 
principalmente utilizando-se ovinos e caprinos, também contaram com seis animais por grupo experimental (FURTADO, 2006; POMROY \& ADLINGTON, 2006).

Segundo SILVA et al. (2003), outros fatores devem ser levados em consideração quando a atividade medicinal de uma planta está sendo analisada, como, por exemplo, a dose total administrada e o período de jejum antes da administração. No presente estudo, os animais foram mantidos em jejum por, pelo menos, 15 horas antes de receberem os tratamentos, tempo considerado suficiente para facilitar a propagação da substância testada através do trato gastrintestinal. Por outro lado, o efeito in vivo provavelmente poderia ser potencializado pelo aumento da dose ou pela repetição da dose por mais tempo. Isso poderia aumentar o tempo de contato entre o hidrolato e o parasito e, presumivelmente, aumentar a redução na fecundidade destes (EGUALE et al., 2007). Entretanto, o aumento na dose pode causar efeitos tóxicos nos hospedeiros e por isso deve ser feito com cautela (ANTHONY et al., 2005).

\section{CONCLUSÕES}

O hidrolato de Mentha villosa Huds. mostrou-se efetivo no controle de larvas de nematóides gastrintestinais de bovinos em teste in vitro. Essa substância não causou uma redução na contagem de opg em bezerras infectadas por nematóides gastrintestinais na dosagem utilizada, quando administrada oralmente aos animais durante 10 dias. A partir dos resultados obtidos, outras metodologias poderão ser utilizadas em busca da validação do efeito anti-helmíntico de $\boldsymbol{M}$. villosa em ruminantes, bem como em outros animais.

\section{COMITÊ DE ÉTICA E BIOSSEGURANÇA}

Este experimento foi aprovado pela Comissão de Ética na Experimentação Animal da Pró-Reitoria de Pesquisa/ UFJF (protocolo: 054/2006-CEA).

\section{REFERÊNCIAS}

ANTHONY, J.P. et al. Plant active components - a resource for antiparasitic agents? Trends in Parasitology, v.21, n.10, p.462-468, 2005.

ARRUDA, T. et al. Preliminary study of the antimicrobial activity of Mentha $\boldsymbol{x}$ villosa Hudson essential oil, rotundifolone and its analogues. Brazilian Journal of Pharmacognosy, v.16, n.3, p.307-311, 2006.
ATHANASIADOU, S. et al. Medicinal plants for helminths parasite control: facts and fiction. Animal, v.1, n.9, p.13921400, 2007. Disponível em: http://journals.cambridge.org/ action $/$ displayFulltext type $=6 \&$ fid $=1358444 \&$ jid $=\&$ v o l u m e I d = \& i s s u e I d =0 9 \& a i d = 13584 $40 \&$ bodyId $=\&$ membershipNumber $=\&$ societyETOC ession=\&fulltextType =RA\&fileId=S1751731107000730. Doi: 10.1017/S1751731107000730.

CAMURÇA-VASCONCELOS, A.L.F. Avaliação da atividade anti-helmíntica dos óleos essenciais de Lippia sidoides e Croton zehntneri sobre nematóides gastrintestinais de ovinos. 2006. 83f. Tese (Doutorado em Ciências Veterinárias) - Programa de Pós-graduação em Ciências Veterinárias, Universidade Estadual do Ceará.

CHAGAS, A.C.S. Controle de parasitas utilizando extratos vegetais. Revista Brasileira de Parasitologia Veterinária, v.13, supl.1, p.156-160, 2004.

COLES, G.C. et al. World Association for the Advancement of Veterinary Parasitology (W.A.A.V.P.) methods for the detection of anthelmintic resistence in nematodes of veterinary importance. Veterinary Parasitology, v.44, p.35-44, 1992.

COLES, G.C. et al. The detection of anthelmintic resistence in nematodes of veterinary importance. Veterinary Parasitology, v.136, p.167-185, 2006.

COSTA, C.T.C. Atividade anti-helmíntica de Azadirachta indica A. Juss sobre nematóides gastrintestinais de ovinos. 2004. 60f. Dissertação (Mestrado em Ciências Veterinárias) - Programa de Pós-graduação em Ciências Veterinárias, Universidade Estadual do Ceará.

EGUALE, T. et al. Haemonchus contortus: in vitro and in vivo anthelmintic activity of aqueous and hydro-alcoholic extracts of Hedera helix. Experimental Parasitology, v.116, p.340345, 2007. Disponível em: http://www.sciencedirect.com/ science?_ob=ArticleURL\&_udi=B6WFH-4MYMFYS$1 \&$ _user $=687358 \&$ \&doc $=1 \&$ _fmt $=\&$ \&orig $=$ search $\&$ _sort $=\mathrm{d}$ $\&$ vi e w $=$ c \&_a c c t $=$ C $000037899 \&$ _version $=1 \&$ \& url Versio n $=0$ \&_u s e rid $=687358 \& \mathrm{~m}$ $\mathrm{d} 5=98505 \mathrm{c} 5 \mathrm{a} 277 \mathrm{bfb} 47 \mathrm{e} 815 \mathrm{a} 91 \mathrm{~d} 74 \mathrm{fc} 1362$. Doi: 10.1016/ j.exppara.2007.01.019.

FURTADO, S.K. Alternativas fitoterápicas para o controle da verminose ovina no Estado do Paraná: testes in vitro e in vivo. 2006. 127f. Tese (Doutorado em Ciências) - Curso de Pós-graduação em Agronomia, Universidade Federal do Paraná.

GITHIORI, J.B. et al. Evaluation of anthelmintic properties of some plants used as livestock dewormers against Haemonchus contortus infections in sheep. Parasitology, v.129, p.245253, 2004. Disponível em: http://journals.cambridge.org/action/ displayFulltext ?type $=6 \&$ fid $=238353 \&$ jid $=\&$ volume $I d=$ \&issueId=02\&aid=238352 \&bod yId=\&membershipNumber =\&societyETOCSession=\&fulltextType=RA\&fileId=S0031182004005566. Doi: $10.1017 /$ S0031182004005566.

GITHIORI, J.B. et al. Use of plants in novel approaches for control of gastrointestinal helminths in livestock with emphasis on small ruminants. Veterinary Parasitology, v.139, p.308- 
320, 2006. Disponível em: http://www.sciencedirect.com/ science?_ob=ArticleURL \&_udi=B6TD7-4K1HDY9$3 \&$ _user $=687358 \&$ _rdoc $=1 \&$ \& fmt $=$ \&_orig $=$ search\&_s ort $=\mathrm{d} \&$ vi e w $=$ c \&_a c c t $=$ C $000037899 \&$ _ver s i o $n=1 \&$ _ u r l Vers i o $n=0 \&$ _ u s e ri d $=68$ 7358\&md5=ea6e52b166537017a44574ce33c30ae8. Doi: 10.1016/j.vetpar.2006.04.021

GORDON, H.M.L.; WHITLOCK, H.V. A new technique for counting nematode eggs in sheep faeces. Journal of Commonwealth Scientific and Industrial Organization, v.12, n.1, p.50-52, 1939

KETZIS, J.K. et al. Chenopodium ambrosioides and its essential oil as treatments for Haemonchus contortus and mixed adultnematode infections in goats. Small Ruminant Research, v.44, p.193-200, 2002. Disponível em: http://www.sciencedirect.com/ science?_ob=ArticleURL\&_udi=B6TC5-45F8Y36$1 \& \_$user $=687358 \& \_$rdoc $=1 \&$ fmt $=\&$ \&orig $=$ search\&_sort $=\mathrm{d} \&$ view $=$ c \&_acct $=$ C $000037899 \&$ _version $=1 \&$ \&urlVers ion $=0 \&$ userid $=687358 \& m d 5=6$ da5b06e60ae242163d5867650282a2e. Doi: 10.1016/S0921-4488(02)00047-0.

KETZIS, J.K. et al. Evaluation of efficacy expectations for novel and non-chemical helminth control strategies in ruminants. Veterinary Parasitology, v.139, p.321-335, 2006. Disponível em: http:// www.sciencedirect.com/science?_ob=ArticleURL\&_udi=B6TD74K5JVWM-18_user=687358\&_rdoc $=18 \_f m t=8 \_o r i g=s e a r c h \&$

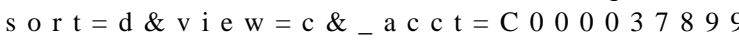
\&__ vers i o n $=1 \&$ \& u r l Versi o $n=0 \&$ userid=687358\&md5=bc2220fd4e3a6c75cdf8807a8b8ab64d. Doi: 10.1016/j.vetpar.2006.04.022

LAHLOU, S. et al. Cardiovascular effects of the essential oil of Mentha $x$ villosa in DOCA-salt-hypertensive rats Phytomedicine, v.9, p.715-720, 2002. Disponível em:http:// www.sciencedirect.com/science? ob=ArticleURL\&_udi=B7GVW4DS3575-CG\&_user $=687358 \& \_$rdoc $=18 \_$fmt $=$\&_orig $=$searc h \&_s ort $=$ d \& vie w = c \& _ a c c t $=$ C 000037899 \& _ version $=1 \&$ \& u r l vers i o $n=0 \&$ _userid $=687358 \& \mathrm{md} 5=$ cb71b4087a81c7f809bc08195e5346d9. Doi: 10.1078/094471102321621313.

MARTINS, A.P. et al. Preparation and characterization of Mentha $\mathrm{x}$ villosa Hudson oil-ß-cyclodextrin complex. Journal of Thermal Analyses and Calorimetry, v.88, n.2, p.363371, 2007.

MEDEIROS, M.F.T. et al. Plantas medicinais e seus usos pelos sitiantes da Reserva Rio das Pedras, Mangaratiba, RJ, Brasil. Acta Botânica Brasilica, v.18, n.2, p.391-399, 2004.

MINISTÉRIO DA AGRICULTURA, PECUÁRIA E ABASTECIMENTO. Portaria no 48, de 12 de maio de 1997. Regulamento técnico para licenciamento e/ou renovação de licença de produtos antiparasitários de uso veterinário. Diário Oficial da União, n.92, de 16 de maio de 1997. p.10165 Seção 1.

MONTE, F.J.Q.; OLIVEIRA, E.F. Triterpenóides pentacíclicos de Mentha $x$ villosa: identificação estrutural e atribuição dos deslocamentos químicos dos átomos de hidrogênio e carbono. Química Nova, v.24, n.4, p.491-500, 2001.
PESSOA, L.M. et al. Anthelmintic activity of essential oil of Ocimum gratissimum Linn. and eugenol against Haemonchus contortus. Veterinary Parasitology, v.109, p.59-63, 2002. Disponível em: http:/ /www.sciencedirect.com/science?_ob=ArticleURL\&_udi=B6TD746RC9HX-1\&_user $=687358 \&$ \& rdoc $=1 \&$ \& $\mathrm{fmt}=\&$ \&o rig $=$ se a r c h \& sort $=$ d \& vie w $=$ c \& a c c t $=C$ $\begin{array}{llllllllll}0 & 0 & 0 & 3 & 7 & 8 & 9 & 9 & \&\end{array}$ - ve r s i o n $=1 \&$ \& u r l V ersion=0\&_userid=687358\&md5=d0bedfa35c24e429e5f127220567bfb1. Doi: 10.1016/S0304-4017(02)00253-4.

POMROY, W.E.; ADLINGTON, B.A. Efficacy of short-term of sulla (Hedysarum coronarium) to young goats against a mixed burden of gastrointestinal nematodes. Veterinary Parasitology, v.136, p.363-366, 2006. Disponível em: http:// www.sciencedirect.com/science?_ob=ArticleURL\&_udi=B6TD7$4 J 2$ VYGN-1\&_user $=687358 \&$ \&doc $=1 \&$ f $\mathrm{mt}=$ \&_orig $=\mathrm{s}$ earch\&_sort $=$ d \& view $=$ c \&_a c ct $=$ C 000037899 $\&$ version $=1 \&$ urlversion $=0 \&$ userid $=687358 \& \mathrm{md} 5=5$ ece13a233a2cffd1b7fcd96350a3721. Doi: 10.1016/j.vetpar.2005.11.018.

RANGEL, V.B. et al. Resistência de Cooperia spp. e Haemonchus spp. às avermectinas em bovinos de corte. Arquivo Brasileiro de Medicina Veterinária e Zootecnia, v.57, n.2, p.186-190, 2005. Disponível em: http://www.scielo.br/scielo.php?script=sci_arttext\&pid=S010209352005000200008\&lng=en\&nrm=iso\&tlng=pt. Doi: 10.1590/ S0102-09352005000200008.

RADÜNZ, L.L. Efeito da temperatura do ar de secagem no teor e na composição dos óleos essenciais de guaco (Mikania glomerata Sprengel) e hortelã-comum (Mentha $\boldsymbol{x}$ villosa Huds). 2004. 90f. Tese (Doutorado em Engenharia agrícola) - Programa de Pós-graduação em Engenharia Agrícola, Universidade Federal de Viçosa.

ROBERTS, F.H.S.; O'SULLIVAN, J.P. Methods of egg counts and larval cultures for strongyles infesting the gastrointestinal tract of cattle. Australian Agricultural Research, v.1, p.99102, 1950.

ROCHFORT, S. et al. Plant bioactives for ruminant health and productivity. Phytochemistry, v.69, p.299-322, 2008. Disponível em: http://www.sciencedirect.com/ science?_ob=ArticleURL\&_udi=B6TH7-4PTN8Y1$1 \&$ \& user $=687358 \&$ \& r oc $=1 \&$ \& $\mathrm{fmt}=\&$ \& orig $=\mathrm{s}$ e arch \&_sort $=$ d \& vi e w $=$ c \& _ a c ct $=$ C 000037 $899 \& \quad$ version $=1$ \& u r l Version $=0$ \& userid $=687358 \& \mathrm{md} 5=9683 \mathrm{a} 0 \mathrm{bd} 61$ baade33896d1f55e6297a2. Doi: $10.1016 /$ j.phytochem.2007.08.017

SILVA, S.L.C. et al. Ação anti-helmíntica de extratos brutos de Andira anthelmia (Vell.) Macbr. e Andira fraxinifolia Benth., em camundongos naturalmente infectados por Vampirolepis nana e Aspiculuris tetraptera.

Latinoamericana, v.58, p.23-29, 2003.

Parasitologia

SOUSA, P.J.C. et al. Effects of piperitenone oxide on the intestinal smooth muscle of the guinea pig. Brazilian Journal of Medical and Biological Research, v.30, n.6, p.787-791, 1997. Disponível em: http://www.scielo.br/scielo.php?script=sci_arttext\&pid=S0100879X1997000600014\&tlng=en\&lng=en\&nrm=iso. Doi: 10.1590/ S0100-879X1997000600014.

SOUZA, A.P. et al. Resistência de helmintos gastrintestinais de bovinos a anti-helmínticos no Planalto Catarinense. Ciência 
Rural, v.38, n.5, p.1363-1367, 2008. Disponível em: http:// www.scielo.br/scielo.php?script $=$ sci_arttext \&pid=S010384782008000500026\&lng=pt\&nrm=iso\&tlng=pt. Doi: 10.1590/ S0103-84782008000500026.

UENO, H.; GONÇALVES, P.C. Manual para diagnóstico das helmintoses de bovinos. 4.ed. Toquio: JIICA, 1998. 143p.

VANDAMME, TH.F.; ELLIS, K.J. Issues and challenges in developing ruminal drug delivery systems. Advanced Drug Delivery Reviews, v.56, p.1415-1436, 2004. Disponível em: h t t p : / / w w w. s c i e n c e d i r e c t. c o m/ science?_ob=ArticleURL\&_udi=B6T3R-4C4W2HN-
$1 \&$ \&user $=687358 \&$ \&doc $=1 \&$ \& fmt $=$ \&_orig $=$ search \&_sort $=\mathrm{d}$ $\&$ vie w $=$ c \&_a c ct $=$ C $000037899 \&$ \& e rsion $=1$ \&_u r l V e r s i o n $=0$ \&_u s e r i d $=68$ 7358\&md5=ceb8338fc6dd790a1ee9bc98034e3f71. Doi: 10.1016/j.addr.2004.02.011

VERCRUYSSE, J. et al. International harmonisation of anthelmintic efficacy guidelines. Veterinary Parasitology, v.96, p.171-193, 2001. Disponível em: http://www.sciencedirect.com/ science?_ob=ArticleURL\&_udi=B6TD 7-42G0KJG$1 \&$ \&user $=687358 \&$ \&doc $=1 \&$ \&mt $=\&$ \&orig $=$ search \&_sort $=\mathrm{d} \&$ view $=$ c\&_acct $=$ C000037899\&_version $=1$ \&_urlVersion=0\&_u serid $=687358 \& m d 5=7 d 7 c 3 e 4 d 1$ ec1c9428a570935f11e3871. Doi: 10.1016/S0304-4017(00)00443-X

Ciência Rural, v.39, n.3, mai-jun, 2009. 